\title{
Interaction of anticancer reduced Schiff base coumarin derivatives with human serum albumin investigated by fluorescence quenching and molecular modeling
}

\author{
Orsolya Dömötör ${ }^{\mathrm{a}}$, Tiziano Tuccinardi ${ }^{\mathrm{b}}$, Darius Karcz ${ }^{\mathrm{c}}$, Maureen Walsh ${ }^{\mathrm{c}}$, Bernadette S. \\ Creaven $^{c}$, Éva A. Enyedy ${ }^{a, d *}$ \\ ${ }^{a}$ Department of Inorganic and Analytical Chemistry, University of Szeged, Dóm tér 7. H-6720 Szeged, Hungary \\ ${ }^{\mathrm{b}}$ Department of Pharmacy, University of Pisa, 56126 Pisa, Italy \\ ${ }^{\mathrm{c}}$ Department of Science, Institute of Technology Tallaght, Dublin 24, Ireland \\ ${ }^{\mathrm{d}}$ HAS-USZ Bioinorganic Chemistry Research Group, Dóm tér 7. H-6720 Szeged, Hungary
}

\begin{abstract}
The specific binding of five reduced Schiff base derived 7-amino-coumarin compounds with antitumor activity to human serum albumin, the principal binding protein of blood, was studied by fluorescence spectroscopy. Their conditional binding constants were computed and the reversible binding at the Sudlow's site I was found to be strong $\left(K_{D} \sim 0.03-2.09 \mu \mathrm{M}\right)$. Based on the data albumin can provide a depot for the compounds and is responsible for their biodistribution and transport processes. The experimental data is complemented by proteinligand docking calculations for two representatives which support the observations. The proton dissociation constants of the compounds were also determined by UV-Vis spectrophotometric and fluorometric titrations to obtain the actual charges and distribution of the species in the various protonation states at physiological $\mathrm{pH}$.
\end{abstract}

Keywords: 7-Amino-coumarin; Human serum albumin; Fluorometry; Dissociation constants; Docking

* Corresponding author: Fax: +36 62433053

E-mail address: enyedy@chem.u-szeged.hu (É.A. Enyedy) 


\section{Introduction}

Human serum albumin (HSA) is the main binding protein for many endogenous and exogenous substances in blood serum. The abundance of HSA in the circulatory system $(0.63$ $\mathrm{mM}, 60 \%$ of the total serum protein level) and its extraordinary acceptor capability with a wide variety of molecules makes it an important tool in the development of novel therapeutic agents, as it influences their pharmacokinetic behavior [1,2]. Thus, HSA is able to increase the in vivo half-life and water solubility of drugs. The deeper knowledge of the binding ability of a drug to the non-specific transporter protein HSA becomes an important research field in chemistry, biochemistry and clinical medicine and screening of the binding to blood proteins is required by the FDA for a potential therapeutic agent [3-6].

HSA is a globular protein, consists of a single chain with 585 amino acids organized in three similar domains (I, II, and III), each consisting of two subdomains (IA, IB, etc.). There are typical sites for binding of several substances such as amino acids, fatty acids, hormones. The principal regions of exogenous drug binding sites are located in hydrophobic pockets in subdomains IIA and IIIA (site I and site II, respectively) [7-10]. Furthermore, the enhanced permeability and retention effect of HSA in cancerous transformed tissues was reported in several studies [11]. Interaction with albumin is of great importance in respect of selective tumor targeting.

Coumarin and its derivatives of both natural and synthetic origins are well known compounds in the food and cosmetic industry, dye- and laser technology, as well as in medicine $[12,13]$. Coumarin compounds are oxygen-containing heterocycles with typical benzopyrone framework. In various pharmacological indications like antibiotic or anticoagulant therapy coumarin derivatives are well proven drugs [14,15]. Furthermore, they have attracted considerable interest as potential antifungal, analgesic, and vasodilator agents in recent years [16-18]. Coumarin derivatives have been also found to be selectively active against cancerous cell lines including malignant melanomas or renal cell carcinomas [19-22]. Halogen-substituted reduced Schiff base coumarin derivatives (1b, 1c, 1d) synthesized by Creaven et al. (Chart 1.) show remarkable anticancer activity against HT29 (human colon cancer) cell line ( $\mathrm{IC}_{50}$ values: $60-82 \mu \mathrm{M}$ ), which was found to be comparable to that of mitoxantrone, an antineoplastic chemotherapy drug [16]. Furthermore the dichloro compound 1b shows additional activity against the MCF-7 (human breast cancer) cell line with an $\mathrm{IC}_{50}$ value of $84 \mu \mathrm{M}[16]$. 
HSA is a well-known transport vehicle of numerous coumarin derivatives [23-25]. Moreover their binding site is site $\mathrm{I}$ in most cases and warfarin as a coumarin derivative anticoagulant drug is also known to bind to the protein at this site [26].

Among optical techniques, fluorescence spectroscopy is an appropriate method to determine the interaction between small ligand molecules and HSA. Coumarin derivatives exhibit strong fluorescence in the visible wavelength region which is usually significantly influenced by the surrounding medium [27]. At the same time HSA contains a single tryptophan (Trp 214) residue near to site I that is responsible for the majority of the intrinsic fluorescence of the protein. This emission can be quenched by a small molecule binding at or near the Trp214 as this amino acid unit is quite susceptible to changes in its environment. Thus the binding at the primarily proposed binding site can be studied directly.

The purpose of the present study is to investigate the binding ability of some reduced Schiff-base coumarin derivatives to HSA using a fluorescence quenching technique, moreover for a deeper understanding of the mechanism of the interaction, proton dissociation $\left(\mathrm{p} K_{a}\right)$ constants were determined for the compounds and the experimental data are complemented by protein-ligand docking calculations.

\section{Chart 1.}

\section{Materials and methods}

\subsection{Chemicals}

Reduced Shiff base derived coumarin compounds (1a, 1b, 1c, 1d, 1e) were synthesized as described previously [16]. HSA (as lyophilized powder with fatty acids), racemic warfarin, $\mathrm{NaH}_{2} \mathrm{PO}_{4}, \mathrm{Na}_{2} \mathrm{HPO}_{4} \mathrm{KOH}, \mathrm{HCl}$ and $\mathrm{NaCl}$ were obtained from Sigma-Aldrich in puriss quality. Doubly distilled Milli-Q water was used for sample preparations.

HSA solution was freshly prepared before the experiments and its concentration was estimated from its UV absorption: $\varepsilon_{280} \mathrm{~nm}(\mathrm{HSA})=36850 \mathrm{M}^{-1} \mathrm{~cm}^{-1}$ [28]. Stock solutions of coumarin derivatives were prepared in dimethyl sulfoxide (DMSO) and were diluted with water or with water containing one equivalent of $\mathrm{NaOH}$ in case of albumin containing samples prior to the analyses.

\section{2. pH-dependent UV-Vis spectrophotometric and spectrofluorometric studies, lipophilicity} predictions 
The $\mathrm{pH}$-dependent measurements for the determination of the proton dissociation constants of the compounds $\mathbf{1 a}, \mathbf{1 b}, \mathbf{1 c}, \mathbf{1 d}, \mathbf{1 e}$ were carried out at $25.0 \pm 0.1{ }^{\circ} \mathrm{C}$ in aqueous phase at a constant ionic strength of $0.10 \mathrm{M}(\mathrm{KCl})$ in the presence of $\leq 0.2 \%(\mathrm{v} / \mathrm{v})$ DMSO content. The titrations were performed with $\mathrm{HCl}(0.10 \mathrm{M})$ and carbonate-free $\mathrm{KOH}$ solution $(0.10 \mathrm{M})$ and their concentrations were determined by $\mathrm{pH}$-potentiometric titrations. $\mathrm{pH}$-Dependent titrations were performed in the $\mathrm{pH}$ range 2.0-11.5. The initial volume of the samples was 20.0 or 30.0 $\mathrm{mL}$. Samples were deoxygenated by bubbling purified argon through them for ca. $10 \mathrm{~min}$ prior the measurements.

The proton dissociation constants and the spectra of the individual species in the various protonation states were calculated with the computer program PSEQUAD [29]. The calculations were always made from the experimental titration data measured in the absence of any precipitate in the solution. A Hewlett Packard 8452A diode array spectrophotometer was used to record the UV-Vis spectra in the interval 200-800 $\mathrm{nm}$. The path length was $4 \mathrm{~cm}$. The spectrophotometric titrations were performed on samples containing $0.5-2.0 \mu \mathrm{M}$ compounds.

The $\mathrm{pH}$-dependent fluorescence measurements were carried out on a Hitachi-4500 spectrofluorometer with the appropriate excitation wavelength and emission wavelength ranges (see details in Table 1). The emission spectra were recorded in $1 \mathrm{~cm}$ quartz cell at 25.0 $\pm 0.1{ }^{\circ} \mathrm{C}$ using $5 \mathrm{~nm} / 5 \mathrm{~nm}$ or $2.5 \mathrm{~nm} / 2.5 \mathrm{~nm}$ slit widths. The samples contained $0.25-1.0 \mu \mathrm{M}$ compound. Three-dimensional spectra were recorded in the 200-450 nm excitation and 210-600 $\mathrm{nm}$ emission wavelength regions at various $\mathrm{pH}$ values.

ClogP values were calculated with the help of ACD/ChemSketch Freeware, version $12.01[30]$.

\subsection{Interaction with HSA: Spectrofluorometric studies}

All samples were prepared in $20 \mathrm{mM}$ phosphate buffer (pH 7.40). Samples contained $1 \mu \mathrm{M}$ HSA and various HSA-to-ligand ratios (from 1:0 to 1:10) were used. Spectra were recorded after $60 \mathrm{~min}$ incubations. The excitation wavelength was $295 \mathrm{~nm}$, the emission intensities were read in the range of 305-550 nm with $5 \mathrm{~nm} / 5 \mathrm{~nm}$ slit widths. The conditional binding constants were calculated with the computer program PSEQUAD [29].

Corrections for self-absorbance and inner filter effect were necessary in the quenching experiments because fluorescence light was significantly absorbed by the coumarinderivatives. The corrections were carried out according to the Equation 1: 


$$
F_{\text {corrected }}=F_{\text {measured }} \times 10^{(A(E X)+A(E M)) / 2} \text { (1) [12] }
$$

where $F_{\text {corrected }}$ and $F_{\text {measured }}$ are the corrected and measured fluorescence intensities, and $A(E X)$ and $A(E M)$ are the absorbance values at the excitation and emission wavelengths of the samples, respectively. The inner filter effect in the case of $\mathrm{pH}$-dependent titrations was negligible and therefore no corrections were applied.

In the site marker displacement experiments, the HSA $(1 \mu \mathrm{M})$ to warfarin ratio was 1:1 and the concentration of compound 1e was varied $(0-3 \mu \mathrm{M})$. Emission spectra were recorded after $1 \mathrm{~h}$ incubation at $310 \mathrm{~nm}$ excitation wavelength with $5 \mathrm{~nm} / 10 \mathrm{~nm}$ slit widths.

\subsection{Molecular modeling studies}

The ligands were built using Maestro [31] and were subjected to a conformational search (CS) of 1000 steps, using a water environment model (generalized-Born/surface-area model) by means of Macromodel [32]. The algorithm used was based on the Monte Carlo method with the MMFFs force field and a distance-dependent dielectric constant of 1.0. The ligands were then energy minimized using the conjugated gradient method until a convergence value of $0.05 \mathrm{kcal} /(\AA \times \mathrm{mol})$ was reached, using the same force field and parameters used for the CS. Automated docking was carried out by means Autodock 4.0 [33]. The X-ray structure of HSA complexed with warfarin (2BXD PDB code [34]) was taken from the Protein Data Bank. The region of interest used by Autodock was defined in order to contain the residues within $10 \AA$ from the original position of warfarin. The ligands were submitted to 30 genetic algorithm runs in the HSA site I and the best ranked pose was taken into account. All the molecular dynamics (MD) simulations were performed using AMBER11 [35] using the parm03 force field at $300 \mathrm{~K}$. The complexes were placed in a rectangular parallelepiped water box. An explicit solvent model for water, TIP3P, was used, and the complexes were solvated with a 12 $\AA$ water cap. Sodium ions were added as counter ions to neutralize the system. Prior to MD simulations, two steps of energy minimization were carried out. In the first stage, we kept the protein and ligand fixed with a position restraint of $100 \mathrm{kcal} /\left(\mathrm{mol} \times \AA^{2}\right)$ and we just energy minimized the positions of the water molecules. In the second stage, we applied a restraint of $10 \mathrm{kcal} /\left(\mathrm{mol} \times \AA^{2}\right)$ only on the $\alpha$ carbons of the receptor. The two energy minimization stages consisted of 10000 steps. The first 1000 steps were Steepest Descent, and the last 9000 were Conjugate Gradient. Molecular dynamics trajectories were run using the energy minimized structure as the input, and particle mesh Ewald electrostatics [36] and periodic boundary conditions were used in the simulation. The time step of the simulations was $2.0 \mathrm{fs}$ with a cutoff of $12 \AA$ for the non-bonded interaction. SHAKE was employed to keep all bonds 
involving hydrogen atoms rigid. Constant-volume periodic boundary MD was carried out for $200 \mathrm{ps}$, during which the temperature was raised from 0 to $300 \mathrm{~K}$. Then $4.8 \mathrm{~ns}$ of constant pressure periodic boundary MD was carried out at $300 \mathrm{~K}$ using the Langevin thermostat to maintain the temperature of our system constant. During the whole simulation, all the $\alpha$ carbons of the protein were blocked with a harmonic force constant of $10 \mathrm{kcal} /\left(\mathrm{mol} \times \AA^{2}\right)$. General Amber force field (GAFF) parameters were assigned to the ligand, while partial charges were calculated using the AM1-BCC method as implemented in the Antechamber suite of AMBER 11.

We extracted from the last $3 \mathrm{~ns}$ of $\mathrm{MD}$ of the ligand-receptor complexes, 300 snapshots (at time intervals of $10 \mathrm{ps}$ ) for each species (complex, receptor and ligand). The various molecular mechanics-Poisson-Boltzmann solvent accessible surface area (MMPBSA) energy terms in Equation 2 were computed as follows.

$$
\mathrm{G}=\text { Gpolar }+ \text { Gnonpolar }+ \text { Emm }- \text { TS (2) }
$$

Electrostatic, van der Waals and internal energies (Emm) were obtained using the SANDER module in AMBER 11. Polar energies (Gpolar) were obtained from the PBSA module of the AMBER 11 program (using the Poisson-Boltzman method) applying dielectric constants of 1 and 80 to represent the gas and water phases, respectively. Nonpolar energies (Gnonpolar) were determined using the MOLSURF program. In order to compare the energetic interactions of the ligands into the protein, we took only the first three terms of Equation 2 into account.

\section{Results and discussion}

\subsection{Proton dissociation processes and calculated lipophilicity of the compounds}

The proton dissociation constants of the compounds 1a, 1b, 1c, 1d, 1e (Chart 1) possessing quite low water solubilities were determined by UV-Vis spectrophotometric as well by fluorometric titrations due to their high intrinsic fluorescence in aqueous solution in the presence of $\leq 0.2 \%(\mathrm{v} / \mathrm{v})$ DMSO. Results are collected in Table 1 .

\section{Table 1}

The proton dissociation constants can be attributed most probably to the deprotonation of the hydroxyl functional group and the process is accompanied by characteristic changes of the ligand bands in the UV-Vis spectra. However, the development of strong bands with 
higher $\lambda_{\max }$ values which results in more extended conjugated $\pi$ electron systems due to the deprotonation could be observed only for 1e (Fig. 1). On the other hand halogen disubstituted compounds display only a moderate shift in $\lambda_{\max }$, moreover in case of $\mathbf{1 a}$ the absorbance changes are minor in the higher wavelength region (Fig. S1 in Supplementary data).

Fig. 1.

Fig. $1 \mathrm{~b}$ shows the increasing absorbance values for $1 \mathrm{e}$ at $410 \mathrm{~nm}$ and the concentration distribution curves calculated with the help of the $\mathrm{p} K_{\mathrm{a}}$ value as a function of $\mathrm{pH}$. The difference between the calculated $\mathrm{p} K_{a}$ values of compounds $\mathbf{1 a}, \mathbf{1 b}, \mathbf{1 c}, \mathbf{1 d}, \mathbf{1 e}$ is in good agreement with literature data considering the trend of their corresponding phenol analogues [37,38]. The halogen substitution in ortho and para positions to the hydroxyl group causes significantly lower $\mathrm{p} K_{a}$ values compared to the unsubstituted derivative 1a owing to the negative inductive effect of the halogen atoms. Moreover the trend $\mathbf{1 b}>\mathbf{1 c}>\mathbf{1 d}$ in the $\mathrm{p} K_{a}$ values can be explained by the decreasing electronegativity of the halogen resulting in a more increased electron delocalization in the aromatic ring, hence more stable conjugated bases can be formed. Nitro substitution in para position increases the acidity of hydroxyl group even more, as this moiety has a strong negative mesomeric effect.

Spectrofluorometry was also applied to determine $\mathrm{p} K_{a}$ of the compounds (Table 1). This technique is especially advantageous in the case of fluorescent compounds with poor water-solubility. In spite of the close structural similarities of the compounds their fluorescence behavior was quite different. The $\mathrm{pH}$-dependent fluorescence emission spectra of 1c are shown in Fig. 2 representing that the initial large intensity of the protonated form (HL) decreases $\sim 38$-fold as it transforms to its deprotonated ( $\mathrm{L}^{-}$) form. During the deprotonation process no drift of the excitation and emission maxima could be observed. A similar behavior can be seen in case of 1a, where there are two excitation maxima with the same emission maximum $(460 \mathrm{~nm})$, one with lower intensity at $240 \mathrm{~nm}$ and the second with higher intensity at $360 \mathrm{~nm}$. Representative 3-dimensional fluorescence spectra of $\mathbf{1 a}$ at $\mathrm{pH} 7.4$ and 11.0 are shown in Fig. 3. On the other hand the deprotonation of $\mathbf{1 b}$ and $\mathbf{1 d}$ is not accompanied by such pronounced intensity decrease (see Fig. S2). Another interesting phenomenon is the unaffected fluorescence spectra of 1e between $\mathrm{pH} 3.3$ and 11.0. It is well known that the electron donating groups (such as amino-group) substituted on coumarin into electrondeficient positions (positions 4, 5 and 7) will increase the intramolecular electron transfer and thus enhance the fluorescence intensity of coumarin derivatives [12,27]. At the same time an electron-deficient 2-hydroxybenzyl substitution on the amino-group can decrease this effect. Accordingly further moderate decrease can be observed by substitution of electron 
withdrawing-group(s) on the 2-hydroxybenzyl moiety. The $\mathrm{p} K_{a}$ values calculated for 1a, 1c and 1d based on the fluorometric titrations are in good agreement with the results of UV-Vis measurements. In case of $\mathbf{1 b}$ the constant arisen from fluorometry is half order of magnitude higher than that obtained by UV-Vis, most probably some non-suspected excited state reactions can shift the acid-base equilibrium [12,39]. It is noteworthy that at $\mathrm{pH}<3.0$ (in case of $1 \mathrm{c}$ at $\mathrm{pH}<5.0$ ) all the studied compounds show significant changes in both UV-Vis and fluorescence emission spectra, which denote the deprotonation step of the amino group. However determination of $\mathrm{p} K_{a}$ values was not feasible from the spectroscopic results. At the same time this process is of no significance, since it does not occur at physiological conditions.

Fig. 2.

Fig. 3.

The presumed strongly lipophilic nature of the studied compounds did not allow us to determine the n-octanol/water partition coefficients $(\log P)$ by the traditional saturation shake flask method. Therefore, calculated lipophilicities $(\mathrm{Clog} P$, Table 1) were predicted from the molecular structures using fragment based prediction with $\mathrm{ACD} / \mathrm{ChemSketch}$ Freeware, version 12.01 [30]. Distribution coefficients $\left(D_{7.4}\right.$, Table 1$)$ were calculated from the partition coefficients with the help of the proton dissociation constants at pH 7.4 [40]. Data reveal the more lipophilic character of the halogen disubstituted derivatives (1b, 1c, 1d), while the nonsubstituted (1a) and nitro substituted (1e) compounds show diminished lipophilicity.

\subsection{Interaction with HSA: spectrofluorometric studies}

It is well known, that coumarin derivatives bind to HSA principally in the hydrophobic binding pocket site I [23-25], therefore first of all the interaction at this site was investigated in detail. HSA contains a single tryptophan (Trp214) residue that is responsible for the majority of the intrinsic fluorescence of the protein. Upon excitation at $295 \mathrm{~nm}$, HSA emits strong fluorescence at $350 \mathrm{~nm}$ which can be attenuated by a binding event at, or close to, the Trp214 due to its susceptibility to changes in its environment. Addition of any ligand to HSA can quench its fluorescence via static quenching most probably with moderate to high efficiency, indicating that the conformation of the hydrophobic binding pocket in site $\mathrm{I}$ is significantly affected by the ligand binding. The time dependence of the interaction was studied and a rapid equilibration was found. After 20 minutes incubation no further changes in the fluorescence spectra could be observed (see Fig. S3), therefore a 1 hour incubation time was applied for the quenching experiments. Since the self-absorption of these compounds is 
significant at the wavelength range of the quenching measurements, a correction equation was applied to minimize this effect (see Section 2.3). The conditional stability binding constants were calculated with the computer program PSEQUAD [29] using the spectra at the wavelength range where the overlapping of the emission of HSA and ligand was not significant. Compounds 1c, 1d and 1e show similar ability to bind on site I, the calculated binding constants are of considerable high value and are comparable with the binding constant of bilirubin to HSA $\left(\log K^{\prime}=7.30\right.$ [41]). Moreover the binding constant calculated for 1a also exceeds the binding ability of warfarin to HSA $\left(\log \mathrm{K}^{\prime}=5.58\right.$ [42]), a drug which is known to be one of the most efficient binders to site I. The quenching ability of a number of the derivatives is summarized in Fig. 4. The simulated curves determined from the $\log K^{\prime}$ and "molar emission intensity" values (calculated with PSEQUAD [29], see details in Supplementary data) are in a fairly good agreement to those obtained by experimental data. Note that the saturation of the measurements does not tend to zero intensities; this phenomenon was included in the calculations. A possible explanation of this effect can be the partial quenching of Trp emission instead of complete quenching; moreover HSA contains other less emitting residues like Tyr whose emission is not affected by an interaction on site I.

\section{Fig. 4.}

As the $\mathrm{pH}$-dependent studies demonstrated (vide supra) the protonated compounds display an outstanding fluorescence activity. This feature is preserved in physiological $\mathrm{pH}$ as well, hence according to literature data a significant change in the position and/or intensity of their fluorescence is expected upon binding to HSA [43]. However, the emission intensity of most of the compounds in our study was insensitive to binding to HSA. Only the fluorescence of 1c was altered in the course of interaction with HSA (see Fig. 5). Upon binding to HSA the emission maximum of 1c was shifted to shorter wavelength, but the predicted increase in its intensity did not occur and rather a moderate decrease could be observed. In this manner the observed behavior could not be explained by the general intramolecular charge transfer (ICT) vs. twisted intramolecular charge transfer (TICT) theory, where the ICT state denotes the highly fluorescent form and a fluorometric inactive form belongs to the TICT state. The TICT state results from a twisting of the electron donor amino-group around the N-C bond [44,45], that twisting can be restricted by interaction with HSA [43]. Most probably the relative position of the phenolic ring to the coumarin ring changes upon binding to HSA, which can cause an adverse change in the fluorescence intensity (see Section 3.3.). Besides, the compound 1c was sensitive likewise for the interaction with HSA. Fig. 5 shows a nonlinear increment in the intensity of emission of $1 \mathrm{c}$ at $450 \mathrm{~nm}$. This phenomenon allowed us to 
calculate binding constant $\log \mathrm{K}^{\prime}=7.54 \pm 0.06$ from the side of the ligand in the wavelength range $400-500 \mathrm{~nm}$, that is far consistent with the constant calculated from the quenching of Trp (see Table 1). This result confirms the assumption that the primary binding site is located in site I of HSA.

Additionally, displacement reactions with the anticoagulant drug warfarin, which is the mostly used and known site marker fluorescence probe for the binding site I of HSA [46], were carried out in the case of compound 1e, as a representative example. Warfarin has weak fluorescence intensity at its excitation wavelength maximum $(310 \mathrm{~nm})$, while binding to HSA induces a significant increase in the emission intensity (see Fig. S4). As compound 1e does not emit at $\lambda_{\mathrm{EM}}<390 \mathrm{~nm}$ the displacement of warfarin by 1e from this binding pocket of the protein is accompanied by a considerable decrease in the emission intensity as clearly shown in Fig. S4.

A further important observation is the significant increase in the solubility of the compounds in presence of HSA, most likely not exclusively as a result of the binding in site I, but a very fragile interaction on protein surface can ensure a 5-6-fold higher solubility [10].

Fig. 5.

\subsection{Molecular modeling}

In order to further understand and characterize the interaction of the studied compounds with HSA at site I, the compounds were analyzed by means of docking studies into the crystallographic structure of the protein. The ligands were docked into HSA using AUTODOCK 4.0 software [33], and the best pose was analyzed. Fig. 6a shows the interactions of the reference compound warfarin (2BXD PDB code [34]), with the coumarin moiety that interacts into the lipophilic pocket mainly delimited by L262, V265, L284, A285, I288, and T315 and the hydroxyl group that shows H-bond interactions with Y174 and H266. With regards to the phenyl ring it mainly interacts with K223, F235, W238 and A239 whereas the carbonyl oxygen interacts with R246. As shown in Fig. 6b, the docking of compound 1a suggests a completely different orientation for the coumarin moiety with the formation of hydrogen bonds with K223. The benzyl group occupies the lipophilic pocket mainly delimited by L262, V265, L284, A285, I288, and T315 that, as previously described, accommodates the coumarin moiety of warfarin and the 2-hydroxy oxygen forms an H-bond with R281. The docking of the anionic form of 1c highlights a disposition very similar to that observed for compound 1a, with the interaction of the coumarin moiety with K223, the interaction of the 
aryl group into the lipophilic pocket and the phenolate oxygen that shows an ionic interaction with R281.

\section{Fig. 6.}

To confirm the reliability of the docking results, and to make a more precise analysis of the ligand-protein interaction, the HSA-1a and HSA-1c complexes were used as starting structures for $5 \mathrm{~ns}$ of MD simulation. Successively, the MD trajectories were further analyzed through the MM-PBSA method [47], which has shown to accurately estimate the ligandprotein energy interaction [48-50]. This approach averages contributions of gas-phase energies, solvation free energies, and solute entropies calculated for snapshots of the complex molecule as well as the unbound components extracted from MD trajectories according to the procedure fully described in Section 2.4.

As shown in Fig. S5, the complexes seem to be stable during the simulations. By analyzing the root-mean-square deviation (RMSD) of all the heavy atoms from the X-ray structures, we observed an initial increase due to the equilibration of the system, followed, after 1500 ps, by a stabilization of the RMSD value around $1.3 \AA$. Regarding the geometry of the compounds, we analyzed the RMSD of the position of the ligands with respect to the starting structures during the simulations. During the whole MD simulations, the ligands appeared to maintain a disposition similar to that of the starting structures, with an RMSD value between 0.6 and $0.9 \AA$. However, it is noteworthy that these calculations cannot give sufficient information about the conformational changes of the protein's structure upon binding.

With regards to the interactions of the two ligands $\mathbf{1 a}$ and $\mathbf{1 c}$, they showed a disposition which is very similar to the starting docked structures, with the maintenance of the H-bond between the oxygen of the coumarin moiety and K223 and between the phenolic (1a) / phenolate (1c) oxygen and R281 (see Fig. 7).

Fig. 7.

In order to further support the greater strength of the interaction of the phenolate oxygen with R281, to be able to explain the higher activity of compounds $\mathbf{1 c}, \mathbf{1 d}, \mathbf{1 e}, \mathbf{1 b}$; a quantitative evaluation of the interactions between HSA and 1a and 1c during the MD simulations have been carried out. In the Table 2, the MM-PBSA results calculated for each ligand into HSA are reported.

Table 2.

The analysis of the MM-PBSA results highlighted that, with respect to compound 1a and due to a higher electrostatic interaction, compound 1c showed better interaction energy. 
Furthermore, it is also interesting to note that, probably due to the presence of the two bromine atoms, the aryl moiety of $\mathbf{1 c}$ also showed slight higher lipophilic interaction energy. These findings strongly confirm the higher conditional binding constant determined for 1c compared with that of ligand $\mathbf{1 a}$.

\section{Conclusions}

Recently more and more studies report the relevance of serum proteins in therapeutic strategies, since all of the systemic administered drugs are transported by the blood and its first encounter is not only with a number of low-molecular weight compounds and various cellular components, but also with a multitude of plasma proteins. The accumulation of HSA and HSA-bound drugs in solid tumors as a consequence of enhanced permeability and retention effect can be an operative way of selective tumor targeting. Coumarin derived compounds are used in a wide variety of industrial and medical applications. Lately promising coumarin compounds with various structures have shown potential as antitumor drugs.

Hereby we examined some reduced Schiff base derived coumarin compounds with anticancer activity. Spectrofluorometry, UV-Vis photometry and molecular modeling calculations were performed to explore the interaction between HSA and these compounds with respect to the type and strength of interaction. $\mathrm{p} K_{a}$ values were determined for the compounds in order to reveal the exact distribution of protonated and deprotonated forms at physiological $\mathrm{pH}$. Compound 1a is almost exclusively in its protonated form, however halogen substituted derivatives $\mathbf{1 b}, \mathbf{1 c}, \mathbf{1 d}$ are about $50 \%$ deprotonated at $\mathrm{pH} 7.40$. 1e owning the lowest $\mathrm{p} K_{a}$ is deprotonated in $79 \%$. Fluorescence quenching study with HSA showed a high to extraordinary high binding on site I for $\mathbf{1 a}$ and for the substituted compounds, respectively. The base compound 1a has somewhat higher, while the substituted derivatives have even higher binding affinity to site I compared with the well known coumarin derivative warfarin. Following the emission changes of compound 1c upon binding to HSA certified our assumption that the primary binding site of these compounds is located in site I of the protein. With help of molecular modeling study it was confirmed that these compounds most likely bind in site I in considerably different orientation than warfarin. Furthermore, molecular dynamic calculations pointed out the role of phenolate in development of electrostatic interactions between the small ligands and the protein. 


\section{Supplementary data}

Mathematical background of the calculation of the binding constants, figures for some $\mathrm{pH}$ dependent or time-dependence UV-Vis, fluorescence spectra, MD simulations.

\section{Abbreviations}

CS conformational search

D distribution coefficient

HSA human serum albumin

ICT intramolecular charge transfer

$\lambda_{\mathrm{EM}} \quad$ fluorescence emission wavelength

$\lambda_{\mathrm{EX}} \quad$ fluorescence excitation wavelength

MD molecular dynamics

MM-PBSA molecular mechanics-Poisson-Boltzmann solvent accessible surface area

$P \quad$ partition coefficient

RMSD root-mean-square deviation

TICT twisted intramolecular charge transfer

\section{Acknowledgments}

This work has been supported by the Hungarian Research Foundation OTKA 103905. This research was realized in the frames of TÁMOP 4.2.4. A/2-11-1-2012-0001 „National Excellence Program Elaborating and operating an inland student and researcher personal support system”. This research was also supported by the Technological Sector Research Programme, Strand 1, under the European Social Fund. D.K. gratefully acknowledges the EMBARK fellowship for additional funding. 


\section{References}

[1] T. Peters, All About Albumin: Biochemistry, Genetics and Medical Applications, Academic Press, San Diego, 1996.

[2] F. Kratz, B. Elsadek, J. Control. Release 161 (2012) 429-445.

[3] The U.S. Food and Drug Administration website, retrieved on March 20, 2012, http://www.fda.gov/OHRMS/DOCKETS/98fr/00n-1269-nfr0001-03.pdf

[4] P. Bourassa, I. Hasni, H.A. Tajmir-Riahi, Food Chem. 129 (2011) 1148-1155.

[5] E. Froehlich, J.S. Mandeville, C.J. Jennings, R. Sedaghat-Herati, H.A. Tajmir-Riahi, J. Phys. Chem. B 113 (2009) 6986-6993.

[6] A. Belatika, S. Hotchandania, J. Bariyangab, H.A. Tajmir-Riahi, Eur. J. Med. Chem. 48 (2012) 114-123.

[7] D.C. Carter, J.X. Ho, Adv. Protein Chem. 45 (1994) 153-204.

[8] T. Peters Jr., Adv. Protein Chem. 37 (1985) 161-245.

[9] X.M He, D.C. Carter, Nature 358 (1992) 209-215.

[10] G. Fanali, A. di Masi, V. Trezza, M. Marino, M. Fasano, P. Ascenzi, Mol. Aspects. Med. 33 (2012) 209-290.

[11] F. Kratz, J. Control. Release 132 (2008) 171-183.

[12] J.R. Lakowicz, Principles of Fluorescence Spectroscopy, $3^{\text {rd }}$ ed. Springer Science, New York, (2006).

[13] B.G. Lake, Food Chem. Toxicol. 37 (1999) 423-453.

[14] D.C. Hooper, J.S. Wolfson, G.L. McHugh, M.B. Winters, M.N. Swartz, Antimicrob. Agents Chemother. 22 (1982) 662-671.

[15] U. Galm, S. Heller, S. Shapiro, M. Page, S.M. Li, L. Heide, Antimicrob. Agents Chemother. 48 (2004) 1307-131.

[16] B.S. Creaven, E. Czeglédi, M. Devereux, É.A. Enyedy, A.Foltyn-Arfa Kia, D. Karcz, A. Kellett, S. McClean, N.V. Nagy, A. Noble, A. Rockenbauer, T. Szabó-Plánka, M. Walsh, Dalton Trans. 39 (2010) 10854-10865.

[17] J.R. Hoult, M. Payá, Gen. Pharmacol. 27 (1996) 713-722.

[18] X.M Peng, G.L. Damu, C. Zhou, Current Pharmaceutical Design 19 (2013) 38843930.

[19] U.S. Weber, B. Steffen, C.P. Siegers, Res. Commun. Mol. Pathol. Pharmacol. 99 (1998) 193-206. 
[20] S.P. Pillai, S.R. Menon, L.A. Mitscher, C.A. Pillai, D.M. Shankel, J. Nat. Prod. 62 (1999) 1358-1362.

[21] A. Maucher, M. Kager, E. von Angerer, J. Cancer Res. Clin. Oncol. 119 (1993) 150154.

[22] E. von Angerer, M. Kager, A. Maucher, J. Cancer Res. Clin. Oncol. 120 (1994) S14S16.

[23] K.J. Fehske, U. Schläfer, U. Wollert, W.E. Müller, Mol. Pharmacol. 21 (1982) 387393.

[24] I. Sjöholm, B. Ekman, A. Kober, I. Ljungstedt-Pahlman, B. Seiving, T. Sjödin, Mol. Pharmacol. 16 (1979) 767-777.

[25] A.M. Zatón, J.M. Ferrer, J.C. Ruiz de Gordoa, M.A. Marquinez, Chem. Biol. Interact. 97 (1995) 169-174.

[26] G. Sudlow, D.J. Birkett, D.N. Wade, Mol. Pharmacol. 11 (1975) 824-832.

[27] H. Li, L. Cai, Z. Chen, in: Advances in Chemical Sensors (Ed.: W. Wang), InTech 2012, pp. 121-150.

[28] N.D. Chasteen, J.K. Grady, C.E. Holloway, Inorg. Chem. 25 (1986) 2754-2760.

[29] L. Zékány, I. Nagypál, in: Computational Methods for the Determination of Stability Constants (Ed.: D. L. Leggett), Plenum Press, New York, 1985, pp. 291-353.

[30] ACD/ChemSketch Freeware, version 12.01, Advanced Chemistry Development, Inc., Toronto, ON, Canada, www.acdlabs.com, 2013.

[31] Maestro, version 9.0; In Schrödinger Inc: Portland, OR, 2009.

[32] Macromodel, version 9.7; In Schrödinger Inc: Portland, OR, 2009.

[33] G.M. Morris, R. Huey, W. Lindstrom, M.F. Sanner, R.K. Belew, D.S. Goodsell, A.J. Olson, J. Comput. Chem. 30 (2009) 2785-2791.

[34] J. Ghuman, P.A. Zunszain, I. Petitpas, A.A. Bhattacharya, M. Otagiri, S. Curry, J. Mol. Biol. 353 (2005) 38-52.

[35] D. A. Case, T.A. Darden, T.E. III. Cheatham, C.L. Simmerling,. J. Wang, R.E. Duke, R. Luo, R.C. Walker, W. Zhang, K.M. Merz, B. Roberts,B. Wang, S. Hayik, A. Roitberg, G. Seabra, I. Kolossváry, K.F. Wong, F. Paesani, J. Vanicek, J. Liu, X. Wu, S.R. Brozell, T. Steinbrecher, H. Gohlke, Q. Cai, X. Ye, J. Wang, M.-J. Hsieh, G. Cui, D.R. Roe, D.H. Mathews, M.G. Seetin, C. Sagui, V. Babin, T. Luchko, S. Gusarov, A. Kovalenko, P.A. Kollman, $A M B E R$, version 11; In University of California: San Francisco, CA, 2010. 
[36] U. Essmann, L. Perera, M.L. Berkowitz, T. Darden, H. Lee, L.G. Pedersen, J. Chem. Phys. 103 (1995) 8577-8593.

[37] SCQuery, The IUPAC Stability Constants Database, Academic Software (Version 5.5), Royal Society of Chemistry, 1993-2005.

[38] R.L. Lundblad, F.M . MacDonald, Handbook of Biochemistry and Molecular Biology, $4^{\text {th }}$ ed. CRC Press, 2010.

[39] B. Valeur, Molecular Fluorescence Principles and Applications; Wiley-VCH GmbH, 2001.

[40] É.A. Enyedy, D. Hollender, T. Kiss, J. Pharm. Biomed. Anal. 54 (2011) 1073-1081.

[41] É.A. Enyedy, L. Horváth, A. Hetényi, T. Tuccinardi, C.G. Hartinger, B.K. Keppler, T. Kiss, Bioorg. Med. Chem. 19 (2011) 4202-4210.

[42] O. Dömötör, C.G. Hartinger, A.K. Bytzek, T. Kiss, B.K. Keppler, É.A. Enyedy, J. Biol. Inorg. Chem. 18 (2013) 9-17.

[43] J. Shobini, A.K. Mishra, K. Sandhya, N. Chandra, Spectrochim. Acta A 57 (2001) $1133-1147$.

[44] J.M. Hicks, M.T. Vandersall, Z. Babarogic, K.B. Eisenthal, Chem. Phys. Lett. 116 (1985) 18-24.

[45] J.M. Hicks, M.T. Vandersall, E.V. Sitzmann, K.B. Eisenthal, Chem. Phys. Lett. 135 (1987) 413-420.

[46] D.E. Epps, T.J. Raub, F.J. Kézdy, Anal. Biochem. 227 (1995) 342-350.

[47] B. Kuhn, P.A. Kollman, J. Med. Chem. 43 (2000) 3786-3791.

[48] O.A. Donini, P.A. Kollman, J. Med. Chem. 43 (2000) 4180-4188.

[49] T. Tuccinardi, S. Bertini, A. Martinelli, F. Minutolo, G. Ortore, G. Placanica, G. Prota, S. Rapposelli, K.E. Carlson, J.A. Katzenellenbogen, M. Macchia, J. Med. Chem. 49 (2006) 5001-5012.

[50] T. Tuccinardi, F. Manetti, S. Schenone, A. Martinelli, M. Botta, J. Chem. Inf. Model. 47 (2007) 644-655. 


\section{Table 1.}

Proton dissociation constants $\left(\mathrm{p} K_{\mathrm{a}}\right)$ of the reduced Schiff base coumarin derivatives determined by and UV-Vis spectrophotometry and spectrofluorometry; $\lambda(E X)_{\max }, \lambda(E M)_{\max }(\mathrm{nm})$ and relative fluorescence emission intensity (a.u.) values for the protonated species (HL) determined by spectrofluorometric titrations; calculated distribution and partition coefficients; overall binding constants to HSA on site $\mathrm{I}\left(\log K^{\prime}\right)\left\{t=25^{\circ} \mathrm{C}\right\}$

\begin{tabular}{|c|c|c|c|c|c|}
\hline & $1 \mathrm{a}$ & $1 \mathrm{~b}$ & $1 \mathrm{c}$ & 1d & $1 \mathrm{e}$ \\
\hline $\mathrm{p} K_{a} \mathrm{UV}-\mathrm{V} \mathbf{i s}^{\mathrm{a}}$ & $9.63 \pm 0.02$ & $7.51 \pm 0.01$ & $7.46 \pm 0.01$ & $7.36 \pm 0.02$ & $6.80 \pm 0.01$ \\
\hline $\mathbf{L}_{7.4}^{-}(\%)$ & 1 & 43 & 45 & 51 & 79 \\
\hline $\mathrm{HL}_{7.4}(\%)$ & 99 & 57 & 55 & 49 & 21 \\
\hline $\mathrm{p}_{a}$ Fluorometry $^{\mathrm{a}}$ & $9.71 \pm 0.01$ & $8.00 \pm 0.01$ & $7.55 \pm 0.01$ & $7.35 \pm 0.01$ & - \\
\hline$\lambda(\boldsymbol{E} \boldsymbol{X})_{\max }$ & 357 & 349 & 360 & 345 & 346 \\
\hline Flu $_{\mathrm{HL}}{ }^{\mathbf{b}}$ & $1.00(453)$ & $0.45(443)$ & $0.66(449)$ & $0.83(445)$ & $0.02(443)$ \\
\hline$C \log P^{\mathrm{c}}$ & $2.9 \pm 0.4$ & $4.4 \pm 0.4$ & $4.9 \pm 0.5$ & $5.1 \pm 0.5$ & $3.0 \pm 0.4$ \\
\hline $\log D_{7.4}{ }^{\mathrm{d}}$ & 2.9 & 4.2 & 4.6 & 4.7 & 2.3 \\
\hline $\log K^{\prime}(\mathrm{HSA})^{\mathrm{e}}$ & $5.68 \pm 0.03$ & $6.45 \pm 0.01$ & $7.5 \pm 0.1$ & $7.22 \pm 0.04$ & $7.09 \pm 0.02$ \\
\hline$K_{D}^{f}$ & $2.09 \mu \mathrm{M}$ & $0.35 \mu \mathrm{M}$ & $0.03 \mu \mathrm{M}$ & $0.06 \mu \mathrm{M}$ & $0.08 \mu \mathrm{M}$ \\
\hline
\end{tabular}

Table 2. MM-PBSA results for the 1a and 1c compounds. $\triangle \mathrm{PBSA}$ is the sum of the electrostatic (Ele), van derWaals (VdW), polar (PB) and non-polar (PBSur) solvation free energy. Data are expressed as kcal $\times \mathrm{mol}^{-1}$.

\begin{tabular}{ccc}
\hline & 1a & 1c \\
\hline VdW & -36.4 & -41.9 \\
Ele & -31.3 & -57.2 \\
PB & 51.9 & 64.0 \\
PBsur & -2.9 & -3.1 \\
SPBSA & $-18.7 \pm 3.3$ & $-38.1 \pm 3.0$ \\
\hline
\end{tabular}




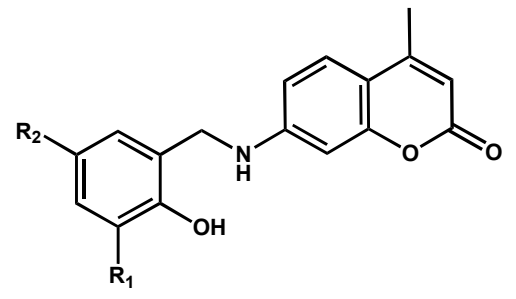

\begin{tabular}{ccc} 
& $\mathbf{R}_{\mathbf{1}}$ & $\mathbf{R}_{\mathbf{2}}$ \\
\hline 1a & $\mathrm{H}$ & $\mathrm{H}$ \\
1b & $\mathrm{Cl}$ & $\mathrm{Cl}$ \\
1c & $\mathrm{Br}$ & $\mathrm{Br}$ \\
1d & $\mathrm{I}$ & $\mathrm{I}$ \\
1e & $\mathrm{H}$ & $\mathrm{NO}_{3}$ \\
\hline
\end{tabular}

Chart 1. Chemical formulae of the studied reduced Schiff base derivatives of coumarin
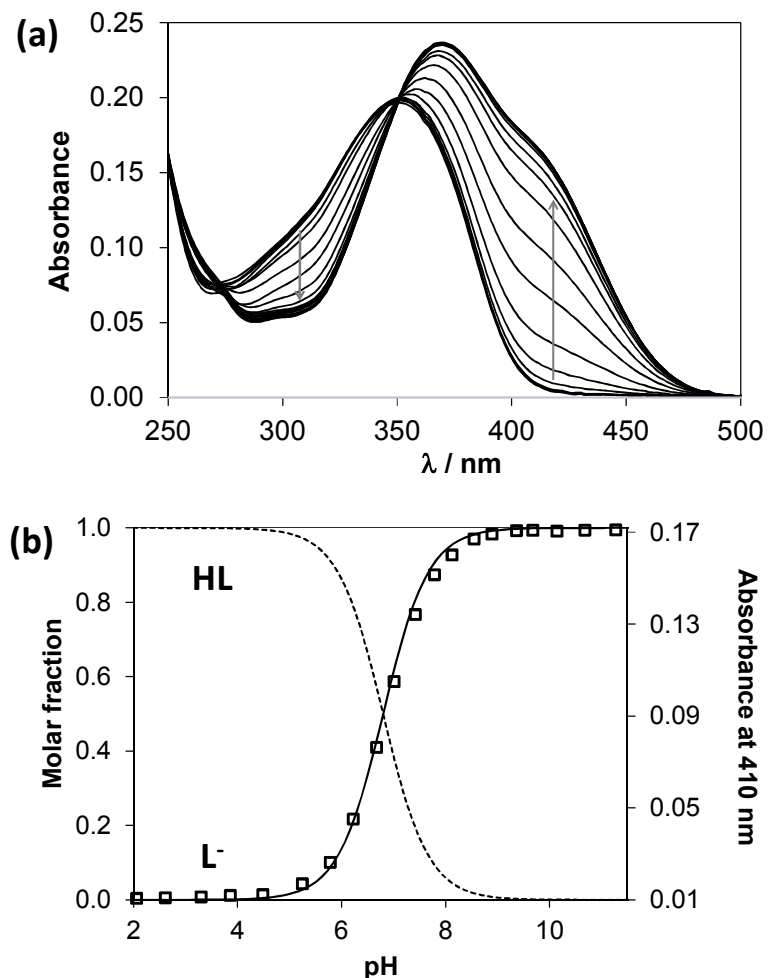

Fig. 1. pH-dependent UV-Vis-spectra of 1e (a), concentration distribution curves and measured absorbance values at $410 \mathrm{~nm}$ (口) as a function of $\mathrm{pH}$ for 1e (b) $\left\{c_{l e}=2 \mu M ; l=4\right.$ $\left.\mathrm{cm} ; t=25^{\circ} \mathrm{C} ; \mathrm{I}=0.1 \mathrm{M}(\mathrm{KCl})\right\}$ 


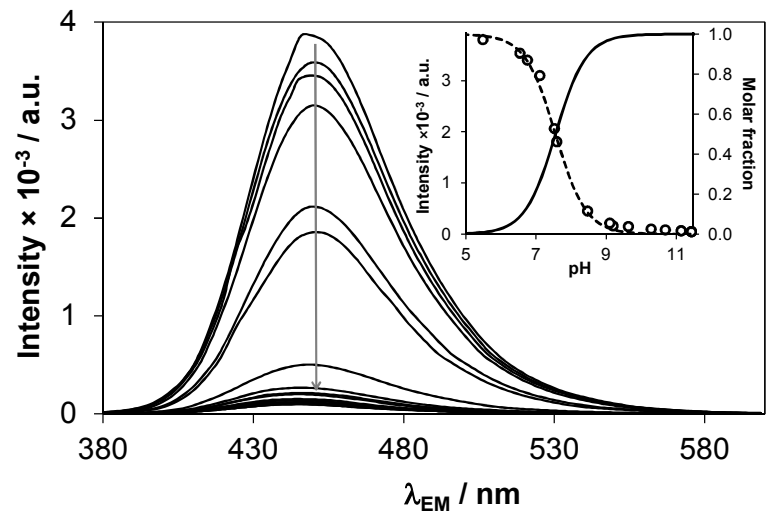

Fig. 2. pH-dependent fluorescence spectra of $\mathbf{1 c}$; inset shows the concentration distribution curves and the $\mathrm{pH}$-dependence of fluorescence at $\lambda_{E M}=450 \mathrm{~nm}\left\{c_{1 c}=0.5 \mu M ; \lambda_{E X}=360 \mathrm{~nm}\right.$; $5 \mathrm{~nm} / 5 \mathrm{~nm}$ slits; $\left.l=1 \mathrm{~cm} ; t=25^{\circ} \mathrm{C} ; I=0.1 \mathrm{M}(\mathrm{KCl})\right\}$
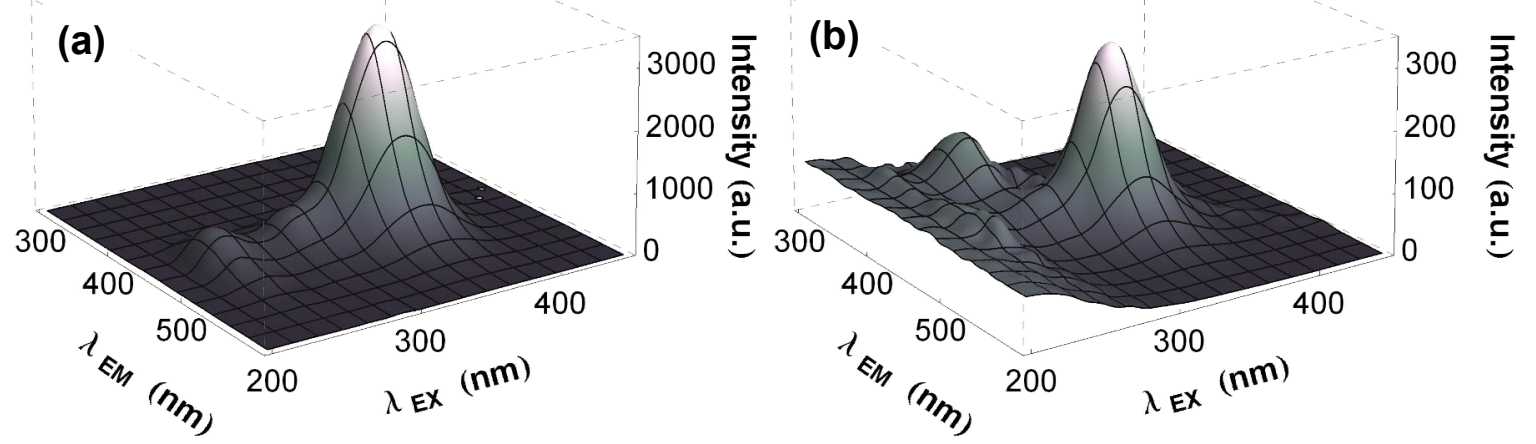

Fig. 3. 3-Dimensional fluorescence spectra of 1 a at $\mathrm{pH} 7.4$ (a) and $\mathrm{pH} 11.0$ (b) $\left\{c_{1 a}=0.5\right.$ $\mu M ; 5 \mathrm{~nm} / 5 \mathrm{~nm}$ slits; $\left.l=1 \mathrm{~cm} ; t=25^{\circ} \mathrm{C} ; I=0.1 \mathrm{M}(\mathrm{KCl})\right\}$ 


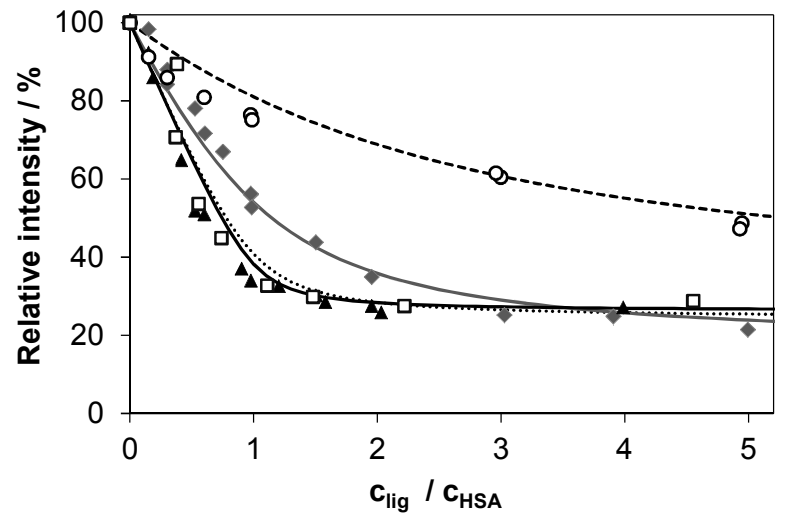

Fig. 4. Measured and calculated quenching of the Trp fluorescence of HSA by the addition of 1a ( $\odot$, black dashed line), $1 \mathbf{b}(\diamond$, grey solid line), 1c ( $\boldsymbol{\Lambda}$, black solid line), or 1d ( $\square$, black dotted line) $\left\{c_{H S A}=1 \mu M ; \lambda_{E X}=295 \mathrm{~nm} ; \lambda_{E M}=342 \mathrm{~nm} ; 5 \mathrm{~nm} / 5 \mathrm{~nm}\right.$ slits; $l=1 \mathrm{~cm} ; t=25$ ${ }^{\circ} \mathrm{C} ; \mathrm{pH}=7.4 ; 20 \mathrm{mM}$ phosphate buffer $\}$

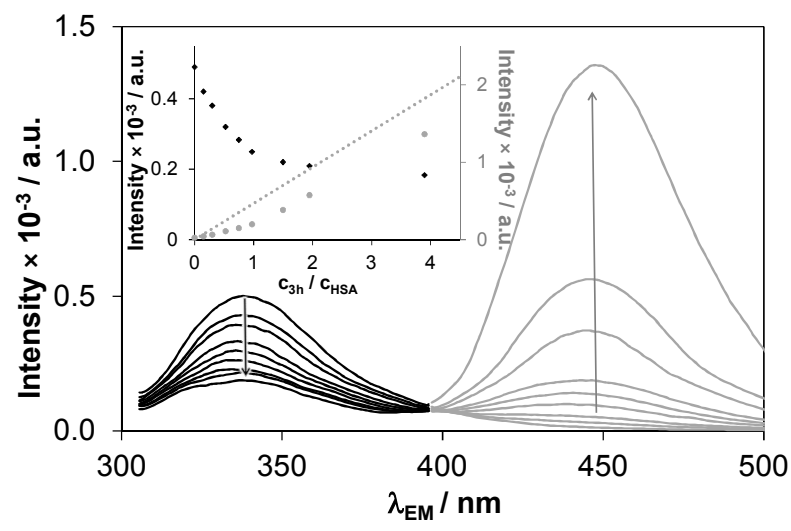

Fig. 5. Fluorescence spectra of HSA-1c system, inset: intensity changes at $340 \mathrm{~nm}$ (black dots) and $450 \mathrm{~nm}$ (grey dots), grey dotted line shows the independent calibration for 1c $\left\{c_{H S A}\right.$ $=1 \mu M ; c_{1 c}=0-4 \mu M ; \lambda_{E X}=295 \mathrm{~nm} ; 5 \mathrm{~nm} / 5 \mathrm{~nm}$ slits; $l=1 \mathrm{~cm} ; t=25{ }^{\circ} \mathrm{C} ; \mathrm{pH}=7.4,20 \mathrm{mM}$ phosphate buffer\} 


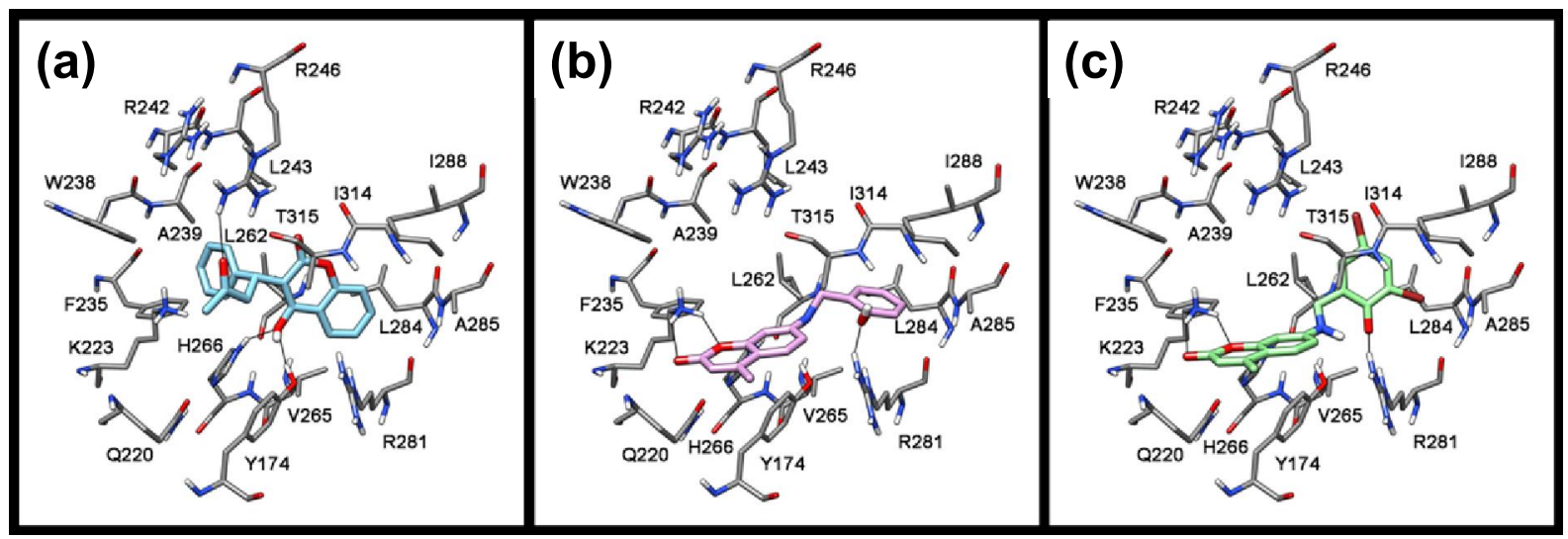

Fig. 6. Analysis of the interaction of warfarin (a) and docking of compound 1a (b) and 1c (c) into the HSA site I.

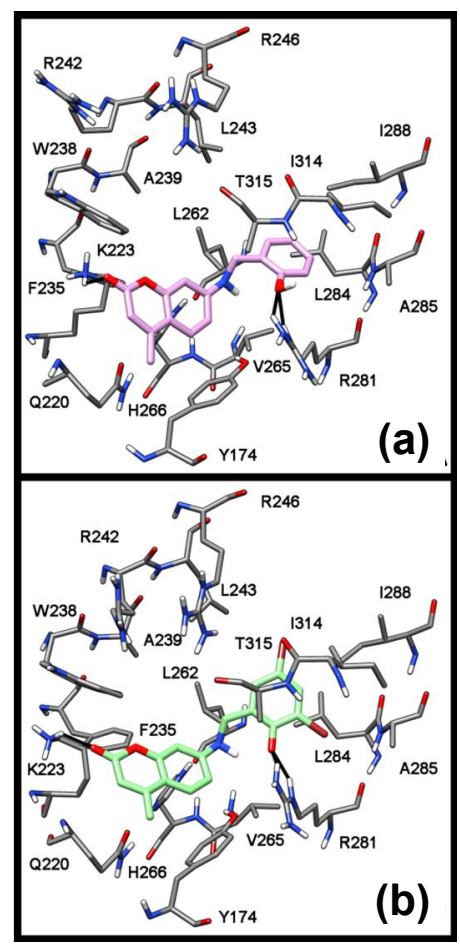

Fig. 7. Minimized average structures resulting from the MD simulation of the 1a-HSA (a) and 1c-HSA (b) complexes. 